\title{
Time-varying pattern of recurrence risk for localized melanoma in China
}

\author{
Xizhi Wen ${ }^{1 \dagger}$, Dandan $\mathrm{Li}^{1 \dagger}$, Jingjing Zhao ${ }^{1}$, Jingjing Li ${ }^{1}$, Tao Yang ${ }^{2}$, Ya Ding ${ }^{1}$, Ruiqing Peng ${ }^{1}$, Baoyan Zhu', \\ Fuxue Huang ${ }^{1}$ and Xiaoshi Zhang ${ }^{1 *}$
}

\begin{abstract}
Background: Acral and mucosal melanomas are rarely seen in Caucasians but common in China. There are limited data on the recurrence characteristics for these patients. This study aimed to identify the recurrence pattern for localized melanoma in China, especially acral and mucosal subtypes.

Methods: Patients with localized melanoma who underwent radical resection between January 1999 and December 2014 in southern China were retrospectively reviewed. Survival and annual recurrence hazard were analyzed by Kaplan-Meier method and hazard function, respectively.

Results: Totally, 1012 patients were included (acral melanoma 400; chronic sun-induced damage (CSD)/non-CSD melanoma 314; mucosal melanoma 298). Recurrence was recorded in 808 patients (localized 14.1\%; regional 29.6\%, and distant 56.3\%). Mucosal melanoma had local and M1c stage recurrence more frequently than cutaneous melanoma, but less frequent regional node relapse. There was no difference in recurrent site distribution between acral and CSD/non-CSD melanoma. The annual recurrence hazard curve for the entire cohort showed a doublepeaked pattern with the first major peak in the second year after surgery and the second peak near the seventh year. Mucosal melanoma had a higher recurrence risk than cutaneous melanoma. Acral melanoma had a lower flat recurrence peak than CSD/non-CSD melanoma. Tumor thickness $>4.0 \mathrm{~mm}$, ulceration, positive regional nodes, and wound infection were associated with a higher recurrence risk in cutaneous melanoma. Adjuvant therapy reduced the recurrence risk of cutaneous melanoma but not of mucosal melanoma.
\end{abstract}

Conclusions: This is a large cohort about the rule of recurrence risk in acral and mucosal melanoma and will provide an initial framework for development of surveillance and adjuvant strategy for Chinese melanoma patients.

Keywords: Melanoma, Recurrence hazard, Chinese population

\section{Introduction}

Malignant melanoma is a heterogenous group of melanocytic neoplasms diagnosed in approximately 20,000 people annually in China [1]. Most patients diagnosed with localized disease are considered curable. However, the risk of loco-regional recurrence or distant metastasis remains high, at between 30 and $60 \%$ in cutaneous melanoma and between 59 and $100 \%$ in mucosal melanoma $[2,3]$. Therefore, understanding the regularity of

\footnotetext{
*Correspondence: 278584385@qq.com; zhangxsh@sysucc.org.cn

${ }^{+}$Xizhi Wen and Dandan Li contributed equally to this work.

${ }^{1}$ Biotherapy Center, State Key Laboratory of Oncology in South China,

Collaborative Innovation Center for Cancer Medicine, Sun Yat-sen University

Cancer Center, Guangzhou 510060, Guangdong, People's Republic of China

Full list of author information is available at the end of the article
}

recurrence is essential for guiding appropriate treatment in localized melanoma patients.

There are significant demographic and ethnic differences in melanoma. In the Chinese population, melanoma arising from the skin accounts for between 50 and $70 \%$, with the acral areas being the most common primary sites. Mucosal melanoma is the second most common subtype, with a percentage incidence of between 22 and 25\% [4]. For Caucasians, approximately $90 \%$ of melanoma arise from the skin, commonly on skin of nonacral sites, while melanoma arising from the mucous membranes and acral areas account for between 1 and 5\% [5]. Acral and mucosal melanomas have been reported to have distinct genetic and clinical characteristics and a poorer prognosis [6-9]. The existing 
melanoma follow-up schedules in China are based on evidence derived from western countries. Postoperative recurrence characteristics of localized melanoma in Chinese patients, especially regarding the acral and mucosal types, are scarce.

In most studies about melanoma, the recurrence risk is analyzed by survival curves but not hazard rates. Compared with survival curves, the hazard rate can reflect both the magnitude of the recurrence rate and its variation with time. The objective of this study was to identify the patterns and high-risk periods of recurrence in Chinese melanoma patients using the hazard function, especially concerning the acral and mucosal melanoma types, and to provide a reference for the designing of follow-up schedules and adjuvant therapy.

\section{Methods}

\section{Patients}

All histologically confirmed malignant melanoma patients who had received radical resection and admitted in Sun Yat-sen University Cancer Center (SYSUCC) between January 1999 and December 2014 were retrospectively analyzed. The patients included in this study met the following criteria: (1) histologically confirmed malignant melanoma; (2) no distant metastasis was found before operation; (3) patients underwent radical surgery; and (4) patients were followed up for at least 3 months. Patients were excluded from the study if they received incomplete resection or neoadjuvant therapy or died of surgical complications. Patients were staged or restaged retrospectively according to the American Joint Committee on Cancer (AJCC) staging system (7th edition). Because sentinel lymph node biopsy is not routinely carried out at our center, only some of the stage I and stage II patients in this study received regional lymph node biopsy. The ultrasonography of regional lymph nodes was routinely carried out as an alternative. Regional lymph node dissection was routinely applied for clinical stage III patients.

\section{Classification of recurrence}

Recurrent sites were categorized into five sites corresponding with AJCC staging criteria, namely: (1) local recurrence; (2) regional nodes, including in transit metastasis; (3) distant skin, soft tissue including muscles, and/or nonregional lymph nodes (M1a); (4) the lungs with or without skin or soft tissue involvement (M1b); and (5) other distant sites, including the liver, gastrointestinal, bone, central nervous system, adrenal glands, eye, and any other sites, and death due to metastases (M1c). If multiple recurrences occurred at the same time, recurrences were counted as one event. A site was classified according to the site with the worst expected prognosis based on staging criteria indicating that non-pulmonary visceral metastases represented the poorest prognostic group, followed by pulmonary metastases, distant skin and soft tissue, and local regional recurrence only.

\section{Statistical analysis}

The statistical methods referred to Zhu JF's study [10]. Recurrence-free survival (RFS) was measured from the time of surgery to the earliest occurrence of relapse (loco-regional or distant), last follow-up, or death from melanoma. Patients who were lost to follow-up or alive at the end of the study were censored for data analysis. RFS was estimated by the Kaplan-Meier method and compared with the log-rank test. For the multivariable analyses, we used two methods to check the proportional hazards (PH) assumption: graphical and timedependent variable approaches. The variables that did not satisfy $\mathrm{PH}$ hypothesis were defined as timedependent variables, which were introduced into the Cox regression model to form Time-Depended Cox Regression Model. The comparison of frequency between each group was performed using the chi-square test. The kernel smoothing method was used to estimate the annual hazard rates and display the graphical display of RFS. A two-sided probability value of less than 0.05 was considered statistically significant. All the statistical analyses were performed using the Stata statistical software package.

\section{Results}

\section{Patient characteristics}

Total of 1012 patients fulfilling the inclusion criteria were included in this study. The median age was 53 years (range, 14-89 years), and the study comprised 534 males and 478 females. Among 714 patients with cutaneous melanoma, 400 were diagnosed with acral melanoma and 314 were diagnosed with melanoma on the skin with chronic sun-induced damage (CSD) or melanoma on skin without chronic sun-induced damage (nonCSD). There were 298 patients with mucosal melanoma included in this study, accounting for $29.4 \%$ of all patients. The most common sites of mucosal melanoma were the head and neck (63.1\%), followed by the genitourinary tract $(21.5 \%)$ and the digestive tract (14.1\%). In addition, there were 4 patients (1.3\%) with mucosal melanoma originating in the respiratory tract. There were 368 cutaneous melanoma patients and 105 mucosal melanoma patients who received adjuvant therapy postoperatively. The clinicopathological characteristics of these patients are detailed in Table 1.

\section{Overall recurrence patterns}

A total of 808 patients (cutaneous melanoma, $n=559$; mucosal melanoma, $n=249$ ) experienced relapse after a 
Table 1 Clinical and pathological characteristics of patients with localized melanoma

\begin{tabular}{lll}
\hline Characteristics & No. & $\%$ \\
\hline Age (years) & & \\
Median & 53 & \\
Range & $14-89$ & \\
Sex & & 52.8 \\
$\quad$ Male & 534 & 47.2 \\
Female & 478 & \\
Clinical subtypes & & 39.5 \\
Acral & 400 & 31.0 \\
CSD/non-CSD & 314 & 29.4 \\
Mucosal & 298 &
\end{tabular}

Anatomic site for mucosal melanoma

$\begin{array}{lll}\text { Head and neck } & 188 & 63.1 \\ \text { Genitourinary tract } & 64 & 21.5 \\ \text { Digestive tract } & 42 & 14.1 \\ \text { Respiratory tract } & 4 & 1.3\end{array}$

AJCC stage (for cutaneous melanoma only)

$\begin{array}{lll}\text { I } & 39 & 5.4 \\ \text { II } & 361 & 50.6 \\ \text { II } & 314 & 44.0\end{array}$

Ulceration (for cutaneous melanoma only)

$\begin{array}{lll}\text { Present } & 342 & 47.9\end{array}$

$\begin{array}{lll}\text { Absent } & 372 & 52.1\end{array}$

Primary tumor thickness (for cutaneous melanoma only)

$\begin{array}{lll}\leq 4.0 \mathrm{~mm} & 228 & 31.9 \\ >4.0 \mathrm{~mm} & 354 & 49.6\end{array}$

Not available

132

18.5

Regional lymph node metastasis (for cutaneous melanoma only) $\begin{array}{lll}\text { Yes } & 400 & 56.0\end{array}$ $\begin{array}{lll}\text { No } & 314 & 44.0\end{array}$

Wound infection after surgery (for cutaneous melanoma only)

$\begin{array}{lll}\text { Yes } & 69 & 9.7 \\ \text { No } & 645 & 90.3\end{array}$

Adjuvant therapy for cutaneous melanoma

Interferon $\mathrm{a}-2 \mathrm{~b} \quad 246 \quad 34.5$

$\begin{array}{lll}\text { Chemotherapy } & 119 & 16.7\end{array}$

$\begin{array}{lll}\text { Radiotherapy } & 3 & 0.4\end{array}$

$\begin{array}{lll}\text { None } & 346 & 48.4\end{array}$

Adjuvant therapy for mucosal melanoma

\begin{tabular}{lll} 
Chemotherapy & 47 & 15.8 \\
Interferon $\mathrm{a}-2 \mathrm{~b}$ & 41 & 13.8 \\
Radiotherapy & 17 & 5.7 \\
None & 193 & 64.8 \\
\hline
\end{tabular}

CSD melanoma on skin with chronic sun-induce damage, non-CSD melanoma on skin without chronic sun-induced damage median follow-up time of 60 months. The first recurrence was diagnosed within 2 years in $81.8 \%$ of the patients, and late recurrences, diagnosed more than 5 years after surgery, were observed in $4.5 \%$ of the patients.

Altogether, 114 (14.1\%) of all first recurrences were classified as local, 239 patients (29.6\%) presented with regional and $455(56.3 \%)$ with distant metastases. The median times to relapse for local, regional, and distant metastases were 8 months, 12 months, and 10 months, respectively. The distribution of recurrence sites was related to pathological types. Compared to cutaneous melanoma, mucosal melanoma had a significantly higher frequency of local recurrence and a lower frequency of regional recurrence (detailed in Table 2). When the distant recurrence was concerned, there were also differences in the distribution of metastasis sites between cutaneous melanoma and mucosal melanoma. The most common metastasis site of mucosal melanoma was other distant site (M1c), followed by the skin or soft tissue (M1a), and finally the lung (M1b). However, for cutaneous melanoma, the most common site of metastasis was the skin/soft tissue (M1a), followed by other distant sites (M1c), and finally the lung (M1b) (Fig. 1). However, there was no statistical difference in the distribution of the recurrence sites between acral and non-acral cutaneous melanoma $(P>0.1)$. The regional lymph node status at diagnosis correlated with the site of recurrence in $\mathrm{cu}$ taneous melanoma. Patients with regional lymph node metastasis at diagnosis were more likely to have distant metastasis. The most common site of recurrence in patients without regional lymph node metastasis at diagnosis was the regional lymph node, followed by distant metastasis (Table 3).

\section{Survival analysis according to clinicopathological factors}

The median RFS for the whole group was 12.0 months. The life-table survival analysis found that the 1-year, 2year, 3-year, 5-year, and 10-year recurrence rates for the whole cohort were $38.0 \%, 28.0 \%, 26.0 \%, 17.0 \%$, and $22.0 \%$, respectively. The median RFS for acral and CSD/ non-CSD melanoma was 16 months and 12 months, respectively. For cutaneous melanoma, the 1-year, 2-year, 3 -year, 5-year, and 10-year recurrence rates were $38 \%$, $26 \%, 24 \%, 17 \%$, and $25 \%$, respectively. For mucosal

Table 2 The relationship between pathological type and the first recurrent sites

\begin{tabular}{llll}
\hline Pathology & \multicolumn{3}{l}{ First recurrent sites } \\
\cline { 2 - 4 } & Local $n(\%)$ & Regional & Distant \\
\hline Cutaneous & $33(5.9 \%)$ & $213(38.1 \%)$ & $313(56.0 \%)$ \\
Mucosal & $81(32.5 \%)$ & $26(10.4 \%)$ & $142(57.0 \%)$ \\
Total & $114(14.1 \%)$ & $239(29.6 \%)$ & $455(5.3 \%)$ \\
\hline
\end{tabular}




\section{$\mathrm{p}=0.02$}

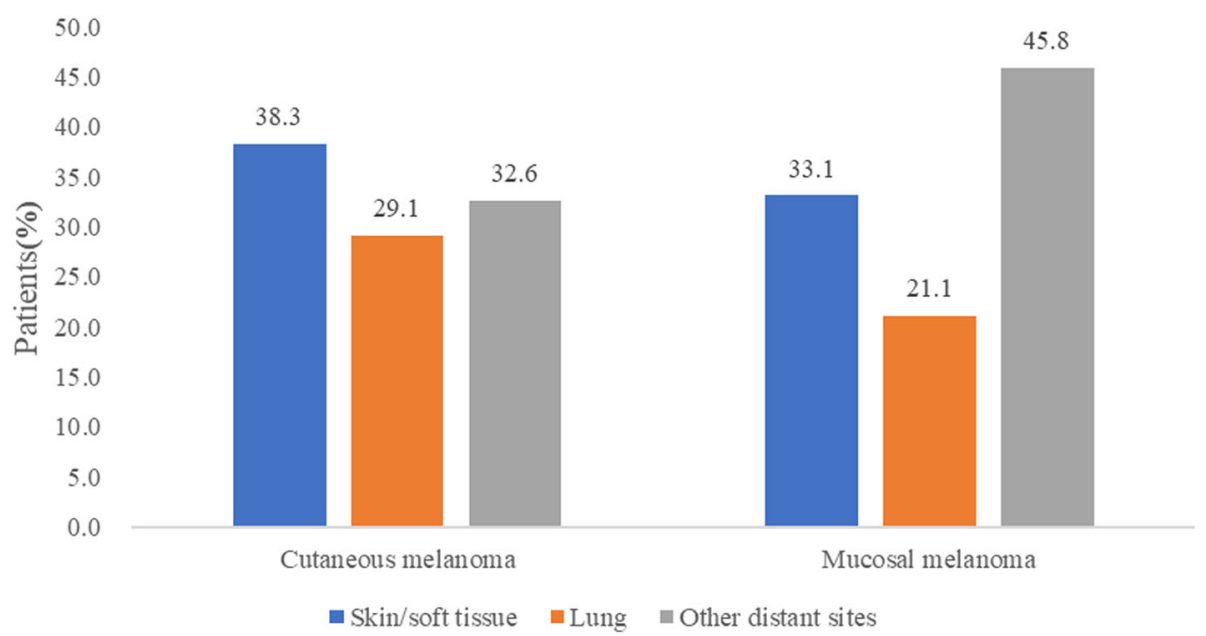

Fig. 1 Comparisons of the frequencies of metastatic sites between cutaneous and mucosal melanoma. The most common metastasis site of mucosal melanoma was other distant sites besides the lung, skin, or soft tissue, but for cutaneous melanoma, the most common site was the skin/soft tissue. $P$ value refers to the chi-square test of the proportion of the metastasis sites for the two groups

melanoma, the median RFS was 11 months, and the 1year, 2-year, 3-year, 5-year, and 10-year recurrence rates were $40.0 \%, 34 \%, 33.0 \%, 18.0 \%$, and $0 \%$, respectively. Both univariable and multivariable analyses revealed that primary non-acral type tumors, tumor thickness greater than $4 \mathrm{~mm}$, primary tumor with ulceration, regional lymph node metastasis, and wound infection postoperatively related to a shorter RFS in cutaneous melanoma. Only lymph node metastasis did not satisfy PH hypothesis with its $\mathrm{HR}$ value changes with time. The $\mathrm{PH}$ assumption test for each variable is listed in Table 4. The Kaplan-Meier survival curve based on each factor is shown in Fig. 2. Adjuvant therapy favored RFS for those with cutaneous melanoma. The anatomic site and postoperative adjuvant therapy, whether chemotherapy, interferon, or radiotherapy, did not relate to the RFS for those with mucosal melanoma. Patient age or sex did not correlate with RFS in the whole cohort. Univariable and multivariable analyses of RFS according to patient's clinicopathological characteristics are listed in Tables 5 and 6.

Table 3 The relationship between regional lymph node status and the first recurrent sites in cutaneous melanoma

\begin{tabular}{llll}
\hline Regional & \multicolumn{2}{l}{ First recurrent sites } \\
\cline { 2 - 4 } $\begin{array}{l}\text { lymph node } \\
\text { at diagnosis }\end{array}$ & Local & Regional lymph node & Distant metastasis \\
\hline Not involved & $25(8.7 \%)$ & $142(49.7 \%)$ & $119(41.6 \%)$ \\
Involved & $8(2.9 \%)$ & $71(26.0 \%)$ & $194(71.1 \%)$ \\
Total & $33(5.9 \%)$ & $213(38.1 \%)$ & $313(56.0 \%)$ \\
\hline
\end{tabular}

\section{Recurrence hazard analysis}

The annual recurrence hazard curve for the entire cohort showed a double-peaked pattern, with a first major recurrence peak at the second year after surgery and covering the first 3 years. The curve then began to fall until the fifth year after surgery, at which point the curves began to rise again, reaching a second peak in the seventh year (Fig. 3).

Subsequently, we compared the recurrence risk concerning different clinicopathological factors, including primary tumor subtype, tumor thickness, ulceration of primary tumor, regional lymph node status, surgical complications (wound infection), and adjuvant therapy. When the hazard rate was related to primary tumor subtype, the analysis showed that mucosal melanoma had a higher recurrence risk than acral or non-acral cutaneous melanoma throughout the observation period. Acral cutaneous melanoma had a lower recurrence risk than non-acral cutaneous melanoma during the first 3 years after surgery and then rose to show a higher recurrence

Table 4 The PH assumption test for variables included in multivariate regression

\begin{tabular}{lll}
\hline Time-dependent covariate & Wald $x^{2}$ & $P$ \\
\hline Primary site *LN(T_) & 3.225 & 0.073 \\
Tumor thickness * $\mathrm{LN}\left(T_{\_}\right)$ & 3.214 & 0.073 \\
Ulceration * $\mathrm{LN}\left(\mathrm{T}_{-}\right)$ & 0.156 & 0.693 \\
Regipnal lymph node metastasis*LN(T_) & 26.93 & $<0.01$ \\
Wound infection * ${ }^{*} \mathrm{~N}\left(\mathrm{~T}_{-}\right)$ & 3.608 & 0.057 \\
Adjuvant therapy* $\mathrm{LN}\left(\mathrm{T}_{-}\right)$ & 0.061 & 0.805 \\
\hline
\end{tabular}




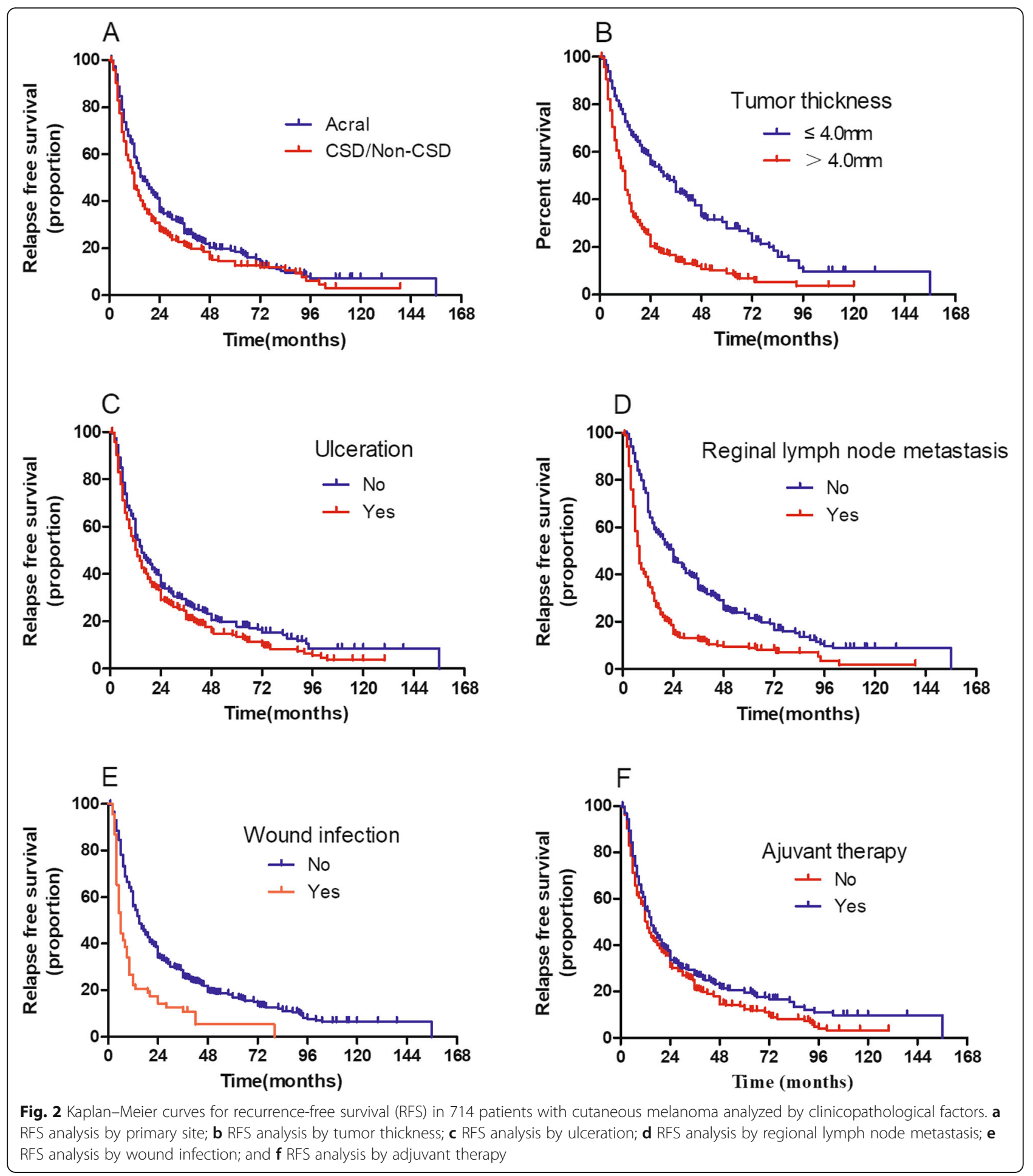

risk in the following 3 to 7 follow-up years and returned to a steady decline afterwards (Fig. 4a).

In the cutaneous melanoma subgroup, the time distribution of the recurrence risk varied with tumor thickness, ulceration, regional lymph node status, and postoperative complications. Patients with primary tumor thickness greater than $4 \mathrm{~mm}$ (T4) demonstrated a more pronounced and variable pattern compared with those with a tumor thickness of $\leq 4 \mathrm{~mm}$ (T1-3). Compared with T4 patients, recurrence peaks for T1-3 patients were relatively flat and late (Fig. 4b). The double-peaked pattern was revealed in both ulcerated and non-ulcerated subgroups with the 
Table 5 Kaplan-Meier postoperative survival analysis (log-rank test) according to clinical-pathological factors

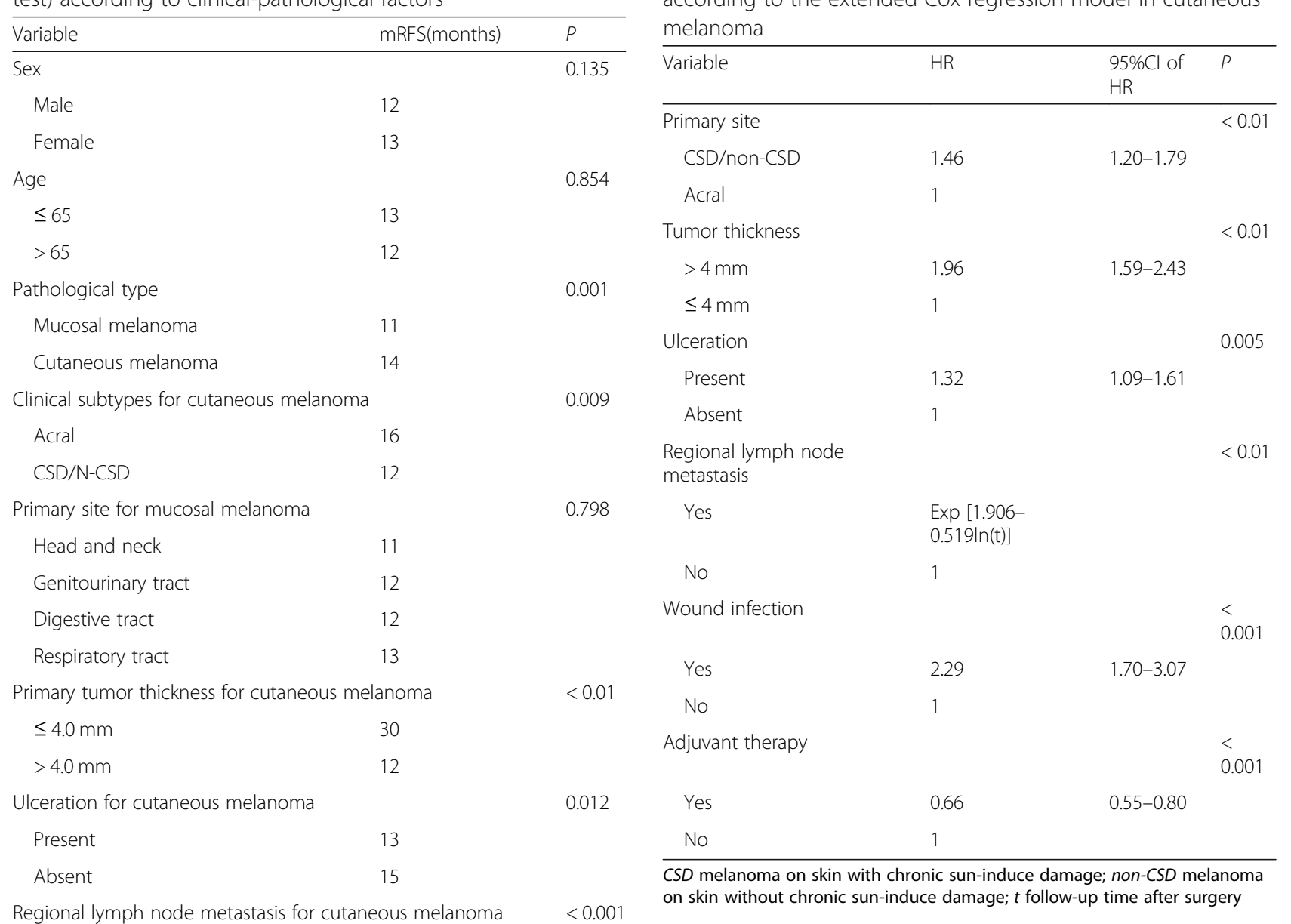

No 24

Stage for cutaneous melanoma

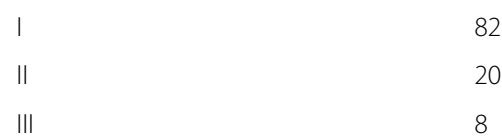

Wound infection for cutaneous melanoma

Yes

No

Adjuvant therapy for cutaneous melanoma

Yes

No

Adjuvant therapy for mucosal melanoma

Yes

No

CSD melanoma on skin with chronic sun-induce damage, non-CSD melanoma

on skin without chronic sun-induced damage

smoothed hazard plots parallel to each other. The annual recurrence hazard curve showed that patients with ulcerative tumors had more recurrences during all analysis
Table 6 Results of multivariable survival analyses for RFS according to the extended Cox regression model in cutaneous melanoma

periods (Fig. 4c). Patients with regional lymph node metastasis at diagnosis revealed taller and steep peaks than patients with negative regional lymph nodes. The hazard curves crossed during the third to the seventh year after surgery (Fig. 4d). Patients who had wound infection after surgery had a markedly higher and earlier recurrence than those without postoperative wound infection (Fig. 4e).

Adjuvant therapy has varying effects on the recurrence curve in different melanoma subtypes. In cutaneous melanoma, the hazard rates for patients receiving adjuvant therapy was lower with the second peak appeared later and flatter than in the non-adjuvant therapy patients (Fig. 4f). In the mucosal melanoma group, adjuvant therapy reduced the magnitude of the two recurrence peaks, with the peaks remaining relatively stable. The hazard curves crossed during the low recurrence risk period (Fig. 4g).

\section{Discussion}

There have been few reports on the time distribution of recurrence hazard rates for acral and mucosal melanomas. In the present study, we compared recurrence 


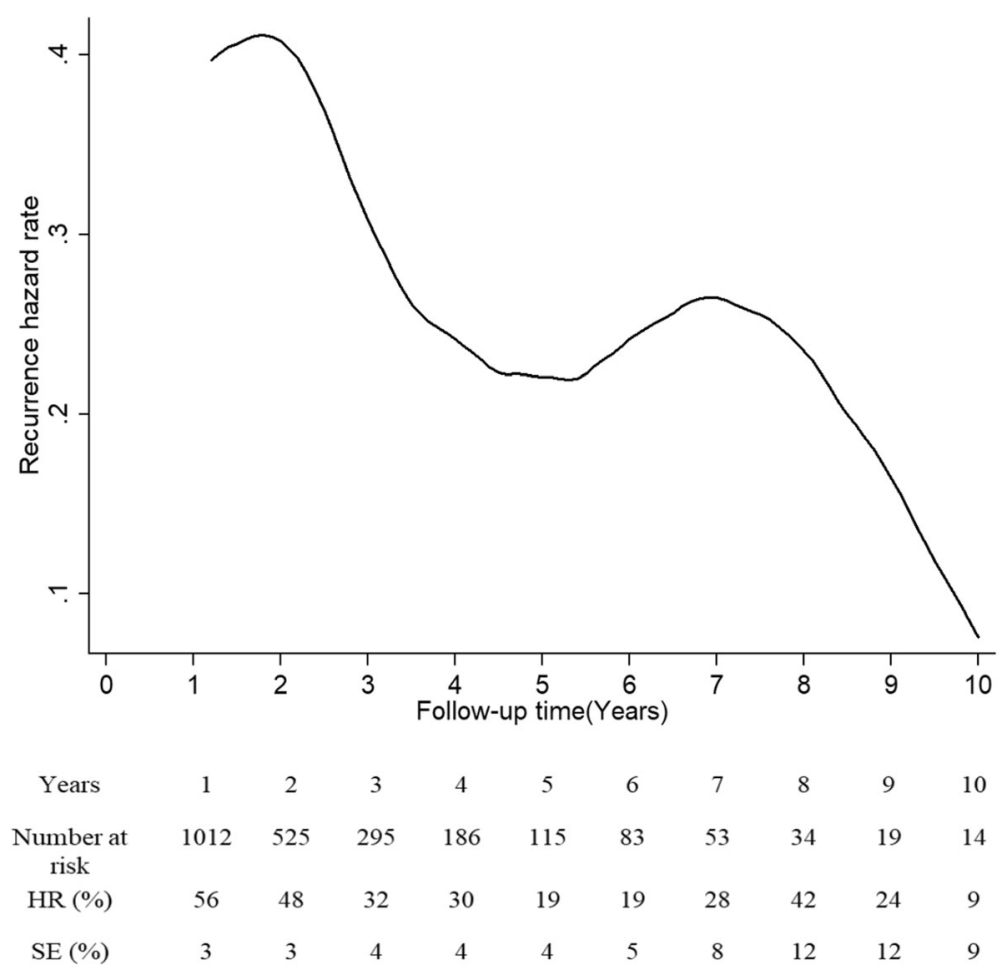

Fig. 3 Recurrent hazard curve for the whole cohort

patterns between different melanoma subtypes and demonstrated the presence of two peaks for recurrence risk among Chinese patients with malignant melanoma, most of whom had acral and mucosal subtypes after complete resection. This pattern has been similarly observed in various tumors such as breast cancer, lung cancer, and gastric cancer [10-12].

The site of recurrence has been reported to be a factor influencing survival after relapse. In our study, we demonstrated that most patients recurred as distant metastases, rather than local or regional recurrences, for both cutaneous and mucosal melanoma, which is similar to findings in another report [13]. In subgroup analysis, we found that mucosal melanoma had a higher frequency of local failure than cutaneous melanoma. This pattern indicated that, aside from systemic adjuvant therapy, local therapy such as radiation therapy $[14,15]$, is also very important for mucosal melanoma. Concerning the pattern of metastatic disease, mucosal melanoma is more likely to metastasize to distant sites other than the skin or the lung, which may explain their short overall survival.

Cutaneous melanoma with regional lymph node involvement at diagnosis had a higher frequency of distant recurrence in our study. Systemic adjuvant therapy should be recommended in preference to local treatment for regional lymph node-positive disease, due to the recurrence pattern of this disease.
Patients with negative regional lymph node involvement at diagnosis showed a higher frequency of local regional recurrence than those with positive regional nodes $(49.7 \%$ vs $26.0 \%)$. The possible reason for this difference is that sentinel lymph node biopsy has not been routinely implemented in our center, which may have resulted in not always detecting clinical occult regional lymph node metastasis. Sentinel lymph node biopsy cannot prolong overall survival [16]. Immediate completion lymph node dissection after positive sentinel lymph node biopsy did not increase melanomaspecific survival but was associated with an increased risk of complications such as wound dehiscence or infection, hematoma, and lymphedema [17]. Therefore, in China, where sentinel lymph node biopsy is not widely promoted, it may be a reasonable option to design an intensified follow-up schedule for regional lymph nodes after resection of the primary lesion for patients with clinically negative regional lymph nodes. On the other hand, we found that patients with wound infection after surgery had a higher recurrence risk and a shorter RFS than those without infection, which suggests that a wider range of surgery needs to be considered carefully.

Immune checkpoint inhibitors have had significant success in treating metastatic melanoma, allowing patients to achieve a long-term survival [18]. Patients with lower tumor burden and good performance are 

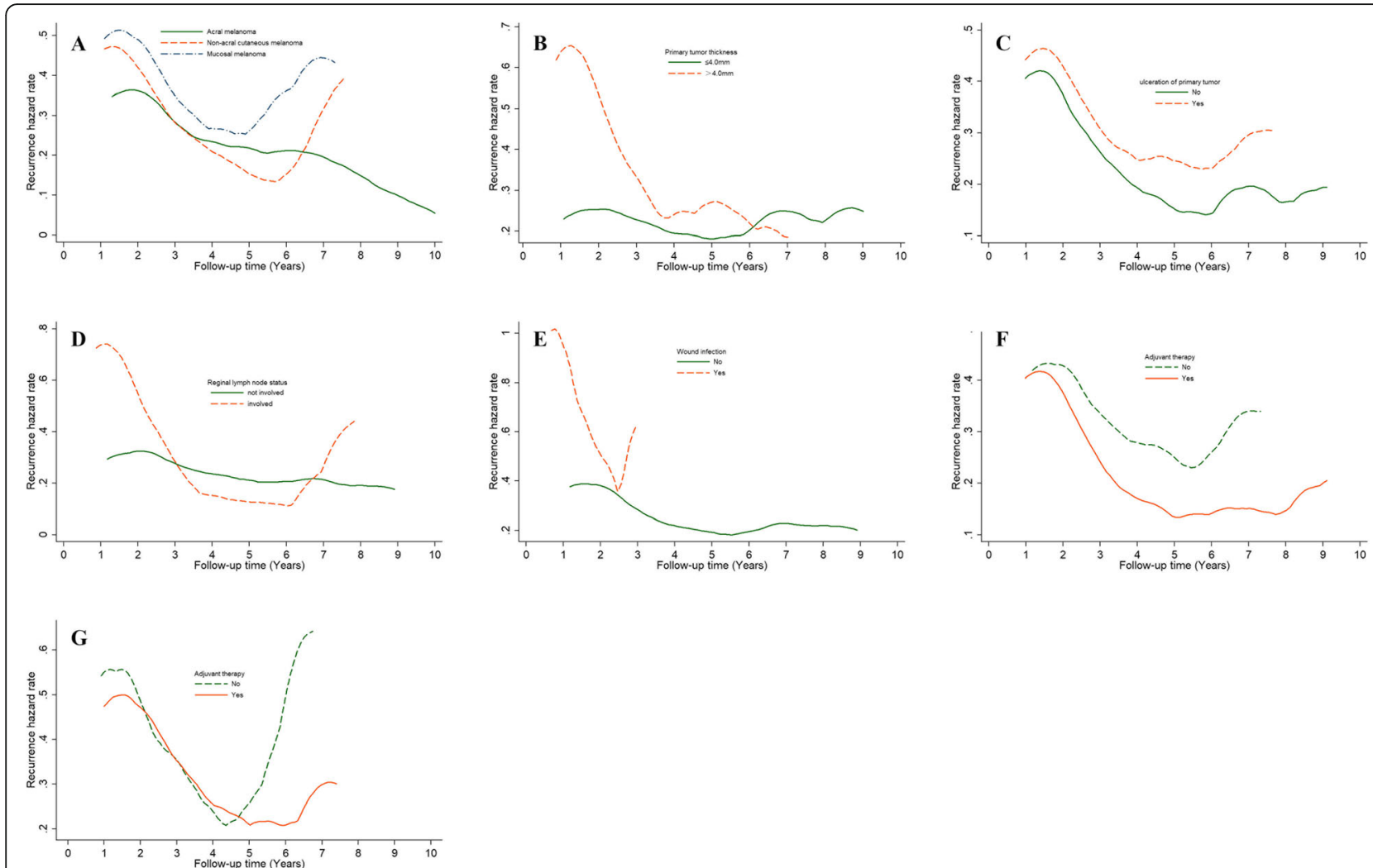

Fig. 4 Recurrence hazard analysis according to clinicopathological characteristics. a pathological types; $\boldsymbol{b}$ primary tumor thickness of cutaneous melanoma; c: ulceration of primary lesion in cutaneous melanoma; $\mathbf{d}$ regional lymph node status for cutaneous melanoma; e wound infection of cutaneous melanoma; $\mathbf{f}$ adjuvant therapy in cutaneous melanoma; $\mathbf{g}$ adjuvant therapy in mucosal melanoma

usually associated with good response rates and longer survival [19]. A structured surveillance program should have a favorable effect on patient prognosis through detecting a recurrence before a patient becomes symptomatic to ensure early treatment. In contrast to melanoma in Caucasians, melanoma in people of Chinese ethnicity manifests as a higher ratio of acral and mucosal types. However, limited data are available regarding the optimal surveillance strategy for patients diagnosed with mucosal or acral melanomas. The existing follow-up schedules for melanoma in the Chinese population are based on previous experiences with Caucasians. Using hazard rate analyses, we found that the risk of initial recurrence in Chinese melanoma patients tended to peak within the first 1 to 2 years after surgery and covered the first 3 years, which is consistent with another report concerning about Caucasians [20]. Our study results support the NCCN guideline recommendation for a routine follow-up every 3 to 6 months for 3 years, and then every 4 to 12 months for two further years [21]. However, it is not recommended to extend the follow-up interval after 5 years, because of the late peak in the seventh year. Additionally, the first recurrent peak involving the first 3 years found in our study supports a long course of adjuvant therapy.

High-risk factors, including ulceration, tumor thickness, and regional lymph node metastasis, are prognostic factors associated with increased risks of recurrence and a shorter survival time among cutaneous melanoma patients [22]. In this study, a double-peaked pattern was observed in a variety of melanoma subgroups. However, for patients with high-risk factors, they exhibited earlier and higher peaks. These factors should be taken into consideration when considering adjuvant therapy and surveillance strategy.

Acral melanoma has been reported to have a worse prognosis than other types of cutaneous melanoma $[6,23]$. Acral melanoma might have a later stage presentation at diagnosis as compared to cutaneous superficial spread melanoma in endemic regions, which may be one of the reasons for its poor prognosis. There are also studies showing that acral melanoma does not differ in terms of patient survival and biological behavior from non-acral melanoma [24]. In the current study, we found that acral melanoma showed a longer RFS and a lower recurrence risk than non-acral cutaneous melanoma. One possible 
explanation for this was that the stage distribution in our series was uneven. There was a lower proportion of stage III disease included in the acral melanoma group in this study $(49.4 \%$ for acral melanoma vs $39.8 \%$ for non-acral cutaneous melanoma). Another explanation was that acral melanoma was reported to have a significantly lower proportion of BRAF mutations than non-acral cutaneous melanoma [25], and BRAF mutation in melanoma was associated with poor prognosis [26], which may lead to a better prognosis for acral melanoma in this study.

\section{Conclusions}

This study involved a large cohort of Chinese melanoma patients with a substantial proportion of acral and mucosal melanoma, providing a preliminary framework for analyzing the patterns and timing of disease recurrence, which is an important step in developing surveillance strategies and adjuvant therapy schedule. Limited by the retrospective design and the lack of routine sentinel lymph node biopsies, further prospective studies are needed to confirm our conclusion.

\section{Abbreviations}

CSD: Chronic sun-induced damageAJCCAmerican Joint Committee on CancerRFSRecurrence-free survivalNCCNThe National Comprehensive Cancer Network

\section{Acknowledgements}

Not applicable.

\section{Authors' contributions}

$X W, D L$, and $X Z$ contributed to the research design. $X W, B Z$, and $Y D$ contributed to the acquisition of the data. XW, JZ, and $J L$ contributed to the analysis and interpretation. XW contributed to the manuscript drafting. TY and $\mathrm{FH}$ gave statistical advice. RP participated in the review of the paper. XZ supervised the research. All authors critically reviewed the manuscript and approved the final revision.

\section{Funding}

This study received support from the National Natural Science Foundation of China (Grant No. 81772910)

\section{Availability of data and materials}

The datasets used or analyzed during the current study are available from the corresponding author on reasonable request.

\section{Ethics approval and consent to participate}

This study was approved by the institutional review board of Sun Yat-sen University Cancer Center and was also conducted in accordance with the Declaration of Helsinki and all patients signed the informed consent.

\section{Consent for publication}

Not applicable.

\section{Competing interests}

The authors declare that they have no competing interests.

\section{Author details}

'Biotherapy Center, State Key Laboratory of Oncology in South China, Collaborative Innovation Center for Cancer Medicine, Sun Yat-sen University Cancer Center, Guangzhou 510060, Guangdong, People's Republic of China. ${ }^{2}$ Department of Orthopedics, Guangdong Provincial People's Hospital,
Guangdong Academy of Medical Sciences, Guangzhou 510060, Guangdong, People's Republic of China.

Received: 23 August 2019 Accepted: 20 December 2019

Published online: 04 January 2020

\section{References}

1. Guo J, Qin S, Liang J, et al. Chinese Guidelines on the Diagnosis and Treatment of Melanoma (2015 Edition). Ann Transl Med. 2015;3(21):322.

2. Balch CM, Gershenwald JE, Soong SJ, et al. Final version of 2009 AJCC melanoma staging and classification. J Clin Oncol. 2009;27(36):6199-206.

3. Lian B, Si L, Cui C, et al. Phase II randomized trial comparing high-dose IFNa2b with temozolomide plus cisplatin as systemic adjuvant therapy for resected mucosal melanoma. Clin Cancer Res. 2013;19(16):4488-98.

4. Chi Z, Li S, Sheng X, et al. Clinical presentation, histology, and prognoses of malignant melanoma in ethnic Chinese: a study of 522 consecutive cases. BMC Cancer. 2011;11:85.

5. McLaughlin CC, Wu XC, Jemal A, Martin HJ, Roche LM, Chen WW. Incidence of noncutaneous melanomas in the U.S. Cancer. 2005;103(5):1000-7.

6. Bradford PT, Goldstein AM, McMaster ML, Tucker MA. Acral lentiginous melanoma: incidence and survival patterns in the United States, 1986-2005. Arch Dermatol. 2009;145(4):427-34.

7. Furney SJ, Turajic S, Stamp G, et al. Genome sequencing of mucosal melanomas reveals that they are driven by distinct mechanisms from cutaneous melanoma. J Pathol. 2013;230(3):261-9.

8. Lian B, Cui CL, Zhou L, et al. The natural history and patterns of metastases from mucosal melanoma: an analysis of 706 prospectively-followed patients. Ann Oncol. 2017;28(4):868-73.

9. Zhou QM, Li W, Zhang X, et al. The mutation profiles of common oncogenes involved in melanoma in southern China. J Invest Dermatol. 2012;132(7):1935-7.

10. Feng $X Y$, Chen $Y B$, Wang $W$, et al. Time-varying pattern of recurrence risk for gastric cancer patients. Med Oncol. 2013;30(2):514.

11. Yin $W, D i G, Z$ hou $L$, et al. Time-varying pattern of recurrence risk for Chinese breast cancer patients. Breast Cancer Res Treat. 2009;114(3):527-35.

12. Zhu JF, Feng $X Y$, Zhang $X W$, et al. Time-varying pattern of postoperative recurrence risk of early-stage (T1a-T2bNOM0) non-small cell lung cancer (NSCLC): results of a single-center study of 994 Chinese patients. PLoS One. 2014:9(9):e106668.

13. Romano E, Scordo M, Dusza SW, Coit DG, Chapman PB. Site and timing of first relapse in stage III melanoma patients: implications for follow-up guidelines. J Clin Oncol. 2010;28(18):3042-7.

14. Lai Y, Meng X, Liu Q, Lu H, Guo L, Wang S, et al. Impact of adjuvant therapy on survival for sinonasal mucosal melanoma. Acta Otolaryngol. 2019:1-6.

15. Yao JJ, Zhang F, Zhang GS, Deng XW, Zhang WJ, Lawrence WR, et al. Efficacy and safety of primary surgery with postoperative radiotherapy in head and neck mucosal melanoma: a single-arm Phase II study. Cancer Manag Res. 2018;10:6985-96.

16. Morton DL, Thompson JF, Cochran AJ, et al. Final trial report of sentinelnode biopsy versus nodal observation in melanoma. N Engl J Med. 2014; 370(7):599-609.

17. Faries MB, Thompson JF, Cochran AJ, et al. Completion dissection or observation for sentinel-node metastasis in melanoma. N Engl J Med. 2017; 376(23):2211-22.

18. Wolchok JD, Chiarion-Sileni V, Gonzalez R, et al. Overall survival with combined nivolumab and ipilimumab in advanced melanoma. N Engl J Med. 2017;377(14):1345-56

19. Ribas A, Hamid O, Daud A, et al. Association of pembrolizumab with tumor response and survival among patients with advanced melanoma. JAMA 2016;315(15):1600-9.

20. Salama AK, de Rosa N, Scheri RP, et al. Hazard-rate analysis and patterns of recurrence in early stage melanoma: moving towards a rationally designed surveillance strategy. PLoS One. 2013;8(3):e57665.

21. Coit DG, Thompson JA, Algazi A, et al. NCCN Guidelines Insights: Melanoma Version 3.2016. J Natl Compr Canc Netw. 2016;14(8):945-58.

22. Gershenwald JE, Scolyer RA, Hess KR, et al. Melanoma staging: evidencebased changes in the American Joint Committee on Cancer eighth edition cancer staging manual. CA Cancer J Clin. 2017;67(6):472-92.

23. Bello DM, Chou JF, Panageas KS, et al. Prognosis of acral melanoma: a series of 281 patients. Ann Surg Oncol. 2013;20(11):3618-25. 
24. Wada M, Ito T, Tsuji G, et al. Acral lentiginous melanoma versus other melanoma: A single-center analysis in Japan. J Dermatol, 2017,44(8):932-938.

25. Si L, Kong Y, Xu X, et al. Prevalence of BRAF V600E mutation in Chinese melanoma patients: large scale analysis of BRAF and NRAS mutations in a 432-case cohort. Eur J Cancer. 2012;48(1):94-100.

26. Houben R, Becker JC, Kappel A, Terheyden P, Bröcker EB, Goetz R, et al. Constitutive activation of the Ras-Raf signaling pathway in metastatic melanoma is associated with poor prognosis. J Carcinog. 2004;3:6.

\section{Publisher's Note}

Springer Nature remains neutral with regard to jurisdictional claims in published maps and institutional affiliations.

Ready to submit your research? Choose BMC and benefit from:

- fast, convenient online submission

- thorough peer review by experienced researchers in your field

- rapid publication on acceptance

- support for research data, including large and complex data types

- gold Open Access which fosters wider collaboration and increased citations

- maximum visibility for your research: over $100 \mathrm{M}$ website views per year

At $\mathrm{BMC}$, research is always in progress.

Learn more biomedcentral.com/submissions 